\title{
LIDT-DD: A New Self-Consistent Debris Disc Model Including Radiation Pressure and Coupling Dynamical and Collisional Evolution
}

\author{
Q. Kral ${ }^{1}$, P. Thebault ${ }^{1}$ and S. Charnoz ${ }^{2}$ \\ ${ }^{1}$ LESIA-Observatoire de Paris, UPMC Univ. Paris 06, Univ. Paris-Diderot, France \\ email: quentin.kral@obspm.fr \\ ${ }^{2}$ Laboratoire AIM, Universite Paris Diderot / CEA / CNRS, Institut Universitaire de France
}

\begin{abstract}
The first attempt at developing a fully self-consistent code coupling dynamics and collisions to study debris discs (Kral et al. 2013) is presented. So far, these two crucial mechanisms were studied separately, with N-body and statistical collisional codes respectively, because of stringent computational constraints.

We present a new model named LIDT-DD which is able to follow over long timescales the coupled evolution of dynamics (including radiation forces) and collisions in a self-consistent way.
\end{abstract}

Keywords. planetary systems: formation stars: circumstellar matter

\section{Context \& Objectives}

Up to now, the evolution of debris discs was studied with two radically different approaches: 1) Dynamics (with N-body codes) to study the structures (e.g. Reche et al. (2008)) and 2) Collisions (with particle-in-a-box codes) to study the grinding process and the resulting collisional cascade in such a disc (e.g. Thébault \& Augereau (2007)). While such separate studies can (and have) produce(d) important results, they suffer from unavoidable limitations. The absence of collisions in N-body codes can indeed strongly bias or even invalidate some results obtained in such approach: As an example, if collisional timescales are shorter than dynamical ones then collisions can hinder or even prevent the build-up of dynamical structures. For the purely statistical models these limitations are obviously the absence of or poor spatial resolution, but also the fact that dynamical processes might strongly affect impact rates and velocities and thus the collisional evolution.

The first attempts at partially coupling dynamics and collisions have been published recently (e.g., CGA and DyCoSS codes by Stark \& Kuchner (2009) and Thebault et al. (2012)), most of them taking as a basis the N-body approach into which some collisionimposed properties are injected. But these codes are limited to steady state cases with only one perturber and no fragmentation.

Our new code LIDT-DD overcomes all these limitations and enables coupling dynamics (with radiation pressure) and collisions in a self-consistent way, and is able to follow complex interactions in discs over long timescales.

\section{The LIDT-DD code: Principle \& First Results...}

LIDT-DD is based on the LIDT3D code developed by Charnoz et al. (2012) for protoplanetary discs, and strongly upgraded to account for the complexity of debris disc physics (high velocity collisions, radiation-pressure affected orbits, wide range of grains' 

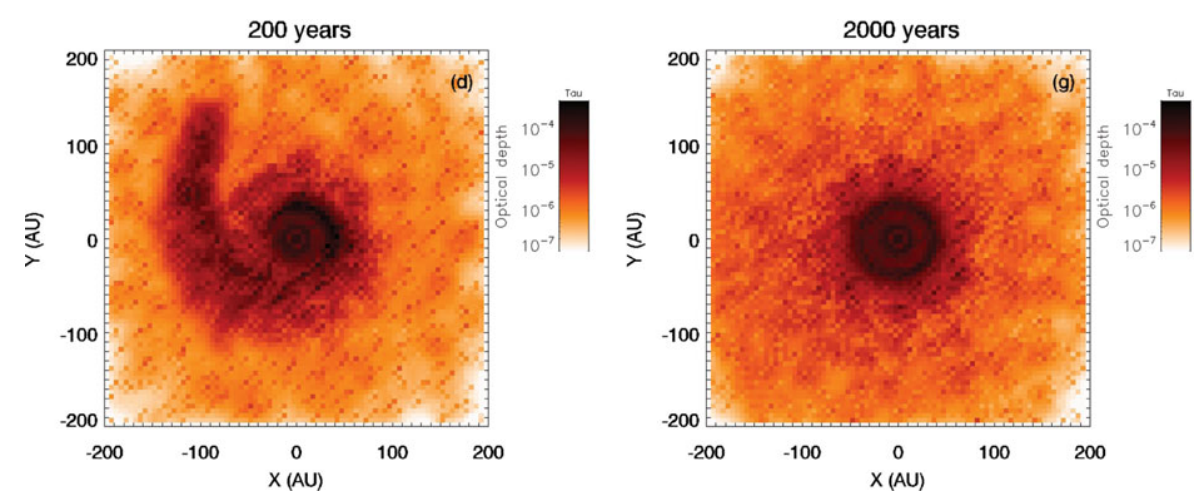

Figure 1. Total smoothed vertical geometrical optical depth evolution in the case of a massive planetesimal breakup within a debris disc.

dynamical behaviour, etc). In this 3D Lagrangian-Eulerian scheme, grains of a given size at a given location in a disc are grouped into "super-particles" (SPs), whose orbits are tracked with an N-body code and whose mutual collisions are treated using a particle-in-a-box scheme. To handle the complexity of grain dynamics, a sorting procedure, regrouping all SPs into dynamical families, has been implemented. A complex SP reassignment routine, looking for and reallocating all redundant SPs, prevents their number from diverging.

Our code has been tested for a set of simplified cases for which we reproduce wellknown robust results. LIDT-DD has also been used on a first astrophysical case: the violent breakup of a massive planetesimal within a debris disc (see Fig 1). Dust due to the breakup is released within a debris disc and its dynamical and collisional evolution is followed. A spiral pattern develops and fades away in 2000 years. A secondary ring is created because of Keplerian shear and survives for millions of years.

\section{Perspectives}

We intend to use LIDT-DD to explore individual systems with "abnormal" flux excesses, such as HD172555 (e.g. Johnson et al. (2012)), and also to investigate how generic the massive-break-up scenario can be for explaining all bright debris discs with luminosities that cannot be explained by classical collisional cascades. Another area of interest for LIDT-DD are the planet-disc interactions and the extent to which planetary companions can sculpt debris discs. LIDT-DD enables the exploration of transient events, multiplanet systems as well as the feedback of the planetary perturbations on the collisional evolution. Another potential application of the code is the puzzling case of bright exozodiacal discs. Scenarios for explaining exozodis, such as massive impacts, planet scattering, falling evaporating bodies, can in principle also be investigated with LIDT-DD.

\section{References}

Charnoz, S. \& Taillifet, E.,2012, ApJ, 753, 119

Johnson, B. C., Lisse, C. M., Chen, C. H., et al. 2012, ApJ, 761, 45

Kral, Q., Thebault, P., \& Charnoz, S. 2013, A\&A, 558, A121

Reche, R., Beust, H., Augereau, J.-C., \& Absil, O. 2008, A\&A, 480, 551

Stark, C. C. \& Kuchner, M. J. 2009, ApJ, 707, 543

Thébault, P. \& Augereau, J.-C. 2007, A\&A, 472, 169

Thebault, P., Kral, Q., \& Ertel, S. 2012, A\&A, 547, A92 